Article Type: Review Article

Subheading: Obstetrics

REVIEW ARTICLE

\title{
A systematic review of primary outcomes and outcome measure reporting in randomized trials evaluating treatments for pre-eclampsia $\downarrow$
}

James M. N. Duffy ${ }^{1,2, \star}$, Martin Hirsch ${ }^{3,4}$, Chris Gale ${ }^{5}$, Louise Pealing ${ }^{1}$, Anusuya Kawsar ${ }^{4}$, Marian Showell ${ }^{6}$, Paula R. Williamson ${ }^{7}$, Khalid S. Khan ${ }^{3}$, Sue Ziebland ${ }^{1}$, Richard J. McManus ${ }^{1}$, on behalf of the International Collaboration to Harmonize Outcomes for Pre-eclampsia (iHOPE)

${ }^{1}$ Nuffield Department of Primary Care Health Sciences, University of Oxford, Oxford, UK

${ }^{2}$ Balliol College, University of Oxford, Oxford, UK

${ }^{3}$ Women's Health Research Unit, Queen Mary, University of London, London, UK

${ }^{4}$ Royal Free London NHS Trust, London, UK

${ }^{5}$ Neonatal Medicine, Faculty of Medicine, Imperial College London, London, UK

${ }^{6}$ Cochrane Gynaecology and Fertility Group, University of Auckland, Auckland, New

Zealand

${ }^{7}$ MRC North West Hub for Trials Methodology Research, Institute of Translational Medicine, University of Liverpool, Liverpool, UK

This article has been accepted for publication and undergone full peer review but has not been through the copyediting, typesetting, pagination and proofreading process, which may lead to differences between this version and the Version of Record. Please cite this article as doi: 10.1002/ijgo.12298

This article is protected by copyright. All rights reserved. 
${ }^{*}$ Correspondence

James M. N. Duffy, Balliol College, University of Oxford, Oxford OX2 6GG, UK.

Email: james.duffy@balliol.ox.ac.uk

Presented as a poster at the Royal College of Obstetricians and Gynaecologists

World Congress; March 20-22, 2017; Cape Town, South Africa. Abstract number: IP162.

This article includes a video abstract, available at https://youtu.be/fcsehou9iUE.

Keywords: Core outcome sets; Maternal; Newborn; Outcome reporting bias; Preeclampsia; Randomized controlled trials; Systematic review

Synopsis: Randomized trials evaluating treatments for pre-eclampsia often omit critical information related to their primary outcome, including definition and measurement. A core outcome set is required.

\begin{abstract}
Background: An evaluation of outcome reporting is required to develop a core outcome set.
\end{abstract}

Objectives: To assess primary outcomes and outcome measure reporting in preeclampsia trials.

Search strategy: Five online databases were searched from inception to January 2016 using terms including "pre-eclampsia" and "randomized controlled trial". Selection criteria: Randomized controlled trials evaluating treatments for preeclampsia published in any language were included. 
Data collection and analysis: Primary outcomes and data on outcome measure reporting were systematically extracted and categorized.

Main results: Overall, 79 randomized trials including data from 31615 women were included. Of those, 38 (48\%) reported 35 different primary outcomes; 28 were maternal outcomes and seven were fetal/neonatal outcomes. Three randomized trials reported composite outcomes, incorporating between six and nine outcome components. The method of definition or measurement was infrequently or poorly reported. Even when outcomes were consistent across trials, different methods of definition or measurement were frequently described.

Conclusions: In randomized trials evaluating interventions for pre-eclampsia, critical information related to the primary outcome, including definition and measurement, is regularly omitted. Developing a core outcome set for pre-eclampsia trials would help to inform primary outcome selection and outcome measure reporting.

\section{INTRODUCTION}

Pre-eclampsia - a pregnancy-specific multisystem syndrome-is a common cause of maternal and neonatal mortality and morbidity [1]. Interventions capable of reducing this substantial health burden are urgently required. Randomized trials are the best way of establishing the efficacy and safety of new treatments, but are only as credible as their primary outcomes [2].

There is currently no consensus regarding the selection of primary outcomes and methods of definition or measurement for pre-eclampsia trials [3]. The primary outcome should be the outcome of greatest therapeutic importance to the study's prospective hypothesis [4]. In the absence of a standardized approach, researchers 
could make arbitrary decisions when choosing among several important outcomes [5]. Within the context of pre-eclampsia, the requirement to evaluate efficacy and safety for the mother and her fetus provides additional complexity. Outcome reporting bias might occur if this selection is made retrospectively on the basis of statistical significance of the results $[6,7]$.

Influenced by factors such as sample size requirement, costs, and time, researchers may need to make pragmatic decisions and select a less informative primary outcome when designing trials [8]. Similarly, researchers may be unable to select otherwise appropriate outcomes owing to a lack of objective definitions or validated instruments. The selection of a composite outcome could increase statistical efficiency owing to higher event rates and can avoid the need to make arbitrary choices among several important outcomes, and would reflect the multisystem nature of pre-eclampsia [9].

The first step in developing a core outcome set for pre-eclampsia requires an evaluation of the reporting of primary outcomes and outcome measures [3]. The aim of the present study was therefore to assess the consistency of primary outcome reporting, including the adequacy of information pertaining to definition and measurement, among randomized trials evaluating treatments for pre-eclampsia.

\section{MATERIALS AND METHODS}

The present systematic review was conducted in accordance with the Preferred Reporting Items for Systematic Reviews and Meta-Analyses (PRISMA) statement 
[10]. A protocol including explicitly defined objectives, study selection criteria, approaches for assessment of study quality, and statistical methods was developed.

The review was undertaken by searching the Cochrane Central Register of Controlled Trials (CENTRAL), Cumulative Index to Nursing and Allied Health Literature (CINHAL), MEDLINE, EMBASE, and PsycINFO, from their inception to January 31, 2016 (Appendix S1). Two researchers (JMND and AK) independently screened each potentially relevant record on the basis of its title and abstract, and then reviewed the full text of each selected study to assess eligibility. Discrepancies between the two researchers were resolved through discussion.

The studies included in the review were randomized controlled trials evaluating the efficacy of any treatment for pre-eclampsia. Trials in mixed populations of prenatal and postnatal women, or in mixed populations of women with pre-eclampsia and chronic hypertension and/or gestational hypertension were excluded. There were no restrictions on language or publication date; two trial reports were translated.

Via a pilot-tested and standardized data extraction form, two researchers independently extracted study characteristics, including participants, interventions, and outcomes. Again, discrepancies between the researchers were resolved by discussion. None of the authors of the trials was contacted to clarify primary outcomes or outcome measures that were unclearly reported.

A comprehensive inventory of primary outcomes was developed. If a primary outcome was not stated explicitly, the outcome included in the study's power 
calculation was extracted. Outcomes were initially organized into two broad categories: maternal outcomes and fetal/neonatal outcomes. These outcomes were then organized into individual domains after consultation with healthcare providers, researchers, and patients.

Descriptive statistics were used to characterize the trials included in the review, mapping primary outcomes and their methods of definition or measurement across included trials. These data were managed in Excel 2013, (Microsoft Corporation, Redmond, WA, USA).

\section{RESULTS}

In total, 7093 titles and abstracts were screened, and 162 potentially relevant studies were examined in detail (Figure 1). Seventy-nine randomized trials, reporting data from 31615 women, met the inclusion criteria. Nearly half these trials $(38 / 79,48 \%)$ reported a primary outcome (Appendix S2).

In total, 35 different primary outcomes were reported, of which 28 were maternal outcomes and seven were fetal/neonatal outcomes. These outcomes were organized in consultation with healthcare professionals, researchers, and patients into 10 domains, including five maternal domains and five fetal/neonatal domains (Table 1).

Primary maternal outcomes that were more frequently reported included blood pressure $(10 / 79,13 \%)$, eclampsia $(7 / 79,9 \%)$, maternal mortality $(3 / 79,4 \%)$, and pulmonary edema $(3 / 79,4 \%)$. Primary fetal/neonatal outcomes were infrequently 
reported: for example, neonatal mortality was reported by $2(3 \%)$ trials, neonatal respiratory distress syndrome was reported by 1 (1\%) trial, and neurologic development by $1(1 \%)$ trial (Table 1$)$.

Three (4\%) trials reported composite outcomes. The number of components ranged from six to nine. Two components—maternal mortality and pulmonary edema—were common to all composite outcomes. One trial included a fetal/neonatal outcome (neonatal repository distress syndrome) within the composite outcomes. In each of the three trial reports, the components of the composite outcome were the same in the abstract, methods, and results.

Six $(8 \%)$ trials reported more than one primary outcome. Three $(3 \%)$ reported more than one primary maternal outcome (range $2-3)$. One (1\%) trial reported two primary fetal/neonatal outcomes. Two (3\%) trials reported primary maternal and fetal/neonatal outcomes (range 2-3).

Thirty-four different methods of definition or measurement were reported (Table 1). Even when outcomes were consistently reported across the trials reviewed, different methods of definition or measurement were described. For example, blood pressure was reported in the following three ways: systolic blood pressure, diastolic blood pressure, and mean arterial blood pressure.

\section{DISCUSSION}

Randomized trials evaluating interventions for pre-eclampsia were found to regularly omit information pertaining to primary outcomes and outcome measures. Nearly half 
of trials in the review explicitly reported a primary outcome. When primary outcomes were consistent across trials, different methods of definition or measurement were frequently described. This variation means that individual studies cannot be compared, contrasted, and combined; the usefulness of research to inform clinical practice is limited.

The strengths of the present systematic review include its originality, comprehensive search strategy, and methodologic design. To our knowledge, the present systematic review is the first to map primary outcomes and their means of definition and measurement in pre-eclampsia trials. To prevent bias in the review process, study selection and data extraction and assessment were conducted independently by two researchers. An international steering group, including women with experience of pre-eclampsia, was formed to oversee the study; their input was central to the development of a comprehensive inventory of primary outcomes.

The empirical evaluation has limitations. Outcomes that were included within a sample size calculation were considered as a primary outcome in the present review. The lack of explicit primary outcomes in many trials meant that outcomes that were not recorded as primary outcomes within the trial report were occasionally mapped. Study authors were not contacted to clarify primary outcomes or outcome measures that appeared unclear. Primary outcomes, especially in earlier-phase efficacy trials, might be chosen to reflect the aim of the intervention; for example, the primary outcome for trials of antihypertensive drugs would not necessarily be expected to be the same as that for trials of anticonvulsants. Examining primary outcome reporting and its relationship with other factors including year of publication, commercial 
funding, and journal impact factor might provide additional understanding [11]; at present, however, no validated tools for quality assessment of outcome reporting exist, limiting the ability to undertake such an analysis.

The Global Pregnancy CoLaboratory—an international collaboration involving key stakeholders including healthcare providers, researchers, and women with experience of pre-eclampsia—have published a strategy to standardize preeclampsia research study design, including data set standards for research studies [12]. Their work reflects the enthusiasm of the pre-eclampsia research community to work together to improve research design and clinical care. The next challenge is to address poor outcome reporting, which drives outcome reporting bias, by developing and implementing core outcome sets.

The Core Outcomes in Women's and Newborn Health (CROWN) initiative has been formed to tackle the challenge of addressing the unwarranted variation in outcome collection and reporting [13]. Participating journals aim to reduce research waste by facilitating consistent reporting of core outcomes [14]. Core outcome sets are minimum collections of outcomes that are predefined, measured in a standardized manner, and reported consistently in the final publication [15]. The outcomes do not need to be extensive and researchers remain free to measure and report other outcomes. Ideally the primary outcome and outcome measure should be selected from the core outcome set. The Core Outcome Measures for Efficacy Trials (COMET) initiative advocates the development of core outcome sets by groups including healthcare providers, researchers, and patients. Their development typically includes three stages: first, identifying potential core outcomes; second, 
determining core outcomes via robust consensus methods among key stakeholders; and third, determining how core outcomes should be measured $[8,15]$. Several consortiums have been established to develop core outcome sets in the obstetrics specialty [16-18].

An international steering group, including healthcare professionals, researchers, and patients, has been formed to develop a core outcome set for pre-eclampsia. The inventory of primary outcomes identified by this systematic review will contribute to the long list of outcomes entered in a modified Delphi method [19]. Consensus "core" outcomes for pre-eclampsia have been identified by 283 healthcare providers, 41 researchers, and 112 patients from 55 countries.

In conclusion, randomized trials evaluating interventions for pre-eclampsia regularly omit information related to the primary outcome and its definition or measurement. Implementing a core outcome set in future pre-eclampsia trials should help to inform primary outcome and outcome measure selection and facilitate consistent reporting.

\section{Author contributions}

JMND, PRW, KSK, SZ, and RJM contributed to study concept and design. JMND, $\mathrm{MH}, \mathrm{LP}, \mathrm{AK}$, and MS contributed to acquisition of data. JMND, MH, CG, PRW, KSK, SZ, and RJM analyzed and interpreted the data. JMND, CG, KSK, SZ, and RJM drafted the manuscript. MH, CG, LP, AK, MS, and PRW revised the manuscript for intellectual content. JMND, PRW, KSK, SZ, and RJM obtained funding. MS and PRW provided administrative, technical, or material support. PRW, KSK, SZ, and 
RJM provided educational supervision. A full description of author contributions is given in Appendix S3.

\section{Acknowledgments}

The study is independent research arising from a doctoral fellowship (DRF-2014-07051) supported by the National Institute for Health Research (NIHR). CG was supported by an NIHR Clinical Trials Fellowship (NIHR-CTF-2014-03-02) and a Medical Research Council Clinician Scientist Fellowship (MR/N008405/1). SZ is a NIHR Senior Investigator. RJM is supported by a NIHR Professorship (NIHR-RP-R212-015) and the NIHR Collaboration for Leadership in Applied Health Research and Care Oxford. The views expressed in this publication are those of the authors and not necessarily those of the National Health Service, the NIHR, or the UK Department of Health. We would like to thank Carla Betts, Dawn Evans, Caroline Jordan, Sam Monaghan, Dan Richards-Doran, Nicola Small, and Clare Wickings at the Nuffield Department of Primary Care Health Sciences, University of Oxford, for administrative, technical, and material support; Tracy Holtham and Rehan Khan at the Women's Health Research Unit, Queen Mary, University of London for administrative, technical support, and subject-specific expertise; and David Mills for administrative and material support.

\section{International Collaboration to Harmonize Outcomes for Pre-eclampsia (iHOPE) steering group}

James M. N. Duffy (University of Oxford, Oxford, UK), Janneke van 't Hooft (Academical Medical Centre, Amsterdam, Netherlands), Chris Gale (Imperial College London, London, UK), Mark Brown (St George and Sutherland Hospitals, Kogarah, 
NSW, Australia), William Grobman (Northwestern University, Chicago, IL, USA), Ray

Fitzpatrick (University of Oxford, Oxford, UK), S. Ananth Karumanchi (Harvard Medical School, Boston, MA, USA), Nuala Lucas (Obstetric Anesthetists' Association, London, UK), Laura Magee (St George's, University of London, London, UK), Ben Mol (University of Adelaide, Adelaide, SA, Australia), Michael Stark (University of Adelaide, Adelaide, SA, Australia), Shakila Thangaratinam (Queen Mary University of London, London, UK), Mathew Wilson (University of Sheffield, Sheffield, UK), Peter von Dadelszen (St George's, University of London, London, UK), Paula R. Williamson (University of Liverpool, Liverpool, UK), Khalid S. Khan (Queen Mary University of London, London, UK), Sue Ziebland (University of Oxford, Oxford, UK), and Richard J. McManus (University of Oxford, Oxford, UK).

\section{Conflicts of interest}

RJM has received blood pressure monitors for research from Lloyds Pharmacies and Omron, and expenses and honoraria for speaking from the American Society of Nephrology and the Japanese Society of Hypertension. The other authors have no conflicts of interest.

\section{References}

[1] Tranquilli AL, Dekker G, Magee L, et al. The classification, diagnosis and management of the hypertensive disorders of pregnancy: A revised statement from the ISSHP. Pregnancy Hypertens. 2014;4(2):97-104.

[2] loannidis JPA, Greenland S, Hlatky MA, et al. Increasing value and reducing waste in research design, conduct, and analysis. Lancet. 2014;383(9912):166-175. 
[3] Duffy JMN, van 't Hooft J, Gale C, et al. A protocol for developing, disseminating, and implementing a core outcome set for pre-eclampsia. Pregnancy Hypertension. $2016 ; 6(4): 274-278$.

[4] Schulz KF, Altman DG, Moher D, et al. CONSORT 2010 statement: updated guidelines for reporting parallel group randomised trials. BMJ. 2010;340:c332. [5] Macefield RC, Boulind CE, Blazeby JM. Selecting and measuring optimal outcomes for randomised controlled trials in surgery. Langenbecks Arch Surg. $2014 ; 399(3): 263-272$.

[6] Saini P, Loke YK, Gamble C, Altman DG, Williamson PR, Kirkham JJ. Selective reporting bias of harm outcomes within studies: findings from a cohort of systematic reviews. BMJ. 2014;349:g6501.

[7] Hutton JL, Williamson PR. Bias in meta-analysis due to outcome variable selection within studies. J Roy Stat Soc. 2000;49:359-370.

[8] Duffy JMN, McManus RJ. Influence of methodology upon the identification of potential core outcomes. Recommendations for core outcome set developers are needed. BJOG. 2016;123(10):1599.

[9] Cordoba G, Schwartz L, Woloshin S, Bae H, Gotzsche PC. Definition, reporting, and interpretation of composite outcomes in clinical trials: systematic review. BMJ. 2010;341:c3920.

[10] Liberati A, Altman DG, Tetzlaff J, et al. The PRISMA statement for reporting systematic reviews and meta-analyses of studies that evaluate health care interventions: explanation and elaboration. J Clin Epidemiol. 2009;62(10):e1-34. [11] Hirsch M, Duffy JMN, Kusznir JO, et al. Variation in outcome reporting in endometriosis trials: a systematic review. Am J Obstet Gynecol. 2016;214(4):452464. 
[12] Myatt L, Redman CW, Staff AC, et al. Strategy for standardization of preeclampsia research study design. Pregnancy Hypertension. 2014;63(6):12931301.

[13] Duffy JMN, Rolph R, Gale C, et al. Core Outcome Sets in Women's and Newborn Health: A Systematic Review. BJOG. 2017;DOI:10.1111/1471-0528.14694. [Epub ahead of print].

[14] Duffy JMN, Bhattacharya S, Herman M, et al. Reducing research waste in benign gynaecology and fertility research. BJOG. 2017;124(3):366-369.

[15] Williamson PR, Altman DG, Blazeby JM, et al. Developing core outcome sets for clinical trials: issues to consider. Trials. 2012;13:132.

[16] Hirsch M, Duffy JMN, Barker C, et al. Protocol for developing, disseminating and implementing a core outcome set for endometriosis. BMJ Open. 2016;6:e13998. [17] Whitehouse KC, Kim CR, Ganatra B, et al. Standardizing abortion research outcomes (STAR): a protocol for developing, disseminating and implementing a core outcome set for medical and surgical abortion. Contraception 2017;95(5):437-441. [18] van 't Hooft J, Duffy JMN, Daly M, et al. A Core Outcome Set for Evaluation of Interventions to Prevent Preterm Birth. Obstet Gynecol. 2016;127(1):49-58. [19] Duffy JMN, Hirsch M, Kawsar A, et al. Outcome reporting across randomised controlled trials evaluating therapeutic interventions for pre-eclampsia: a systematic review. BJOG. 2017;DOI:10.1111/1471-0528.14702. [Epub ahead of print]. 
Figure legend

Figure 1 Flow chart showing the randomized controlled trials included in the review.

Supporting information legends

Appendix S1 EMBASE search strategy.

Appendix S2 Details of the 38 trials for which primary outcomes were reported.

Appendix S3 Full author contributions.

This article is protected by copyright. All rights reserved. 
Table 1 Methods of definition or measurement for primary outcomes reported in pre-eclampsia trials.

Primary outcome definition

No. of randomized

1 Maternal mortality

controlled trials

Not defined

3

2 Maternal morbidity

Hypotension: systolic BP $<40 \mathrm{~mm} \mathrm{Hg}$

Impaired liver function: ACOG 2013 definition

Renal insufficiency: ACOG 2013 definition

Thrombocytopenia

ACOG 2001 definition

Not defined

Pulmonary edema

ACOG 2013 definition

Not defined

Severe pre-eclampsia $>160 / 110 \mathrm{~mm} \mathrm{Hg}$ or proteinuria $5 \mathrm{~g} / 24 \mathrm{~h}$

Unclear

Eclampsia

Witnessed tonic-clonic seizure

Witnessed seizure

Not defined

HELLP syndrome

Platelets $<100 \times 10^{9} / \mathrm{L}, \mathrm{AST}>70 \mathrm{U} / \mathrm{L}, \mathrm{ALT}>70 \mathrm{U} / \mathrm{L}$

Not defined

Placental abruption: not defined

Postpartum hemorrhage: blood loss $>1000 \mathrm{~mL}$ within $24 \mathrm{~h}$ of delivery

Neurologic symptoms: ACOG 2013 definition

3 Labor and delivery characteristics

Pregnancy prolongation: intervention to delivery, $d$

Duration of labor

Intervention to delivery, $\mathrm{h}$

Unclear $^{a}$

Mode of delivery: cesarean delivery (yes/no)

Indication for delivery: recurrent late or severe variable decelerations

4 Therapeutic interventions

Magnesium sulfate infusion duration: unclear $^{\text {a }}$

Magnesium sulfate infusion reinstituted

Not defined

Unclear $^{\text {a }}$

Treatment failure: systolic BP $>90 \mathrm{~mm} \mathrm{Hg}$ post-intervention

1

1

1

1

1

1

1

1

1

3

Maternal biological indices

Blood pressure

Systolic BP

Diastolic BP

Mean arterial pressure

Unclear $^{2}$

Proteinuria: $>5 \mathrm{~g} / 24 \mathrm{~h}$

Cardiac function: cardiac index; thoracic electrical bioimpedance

Heart rate: beats per minute

Renal function: creatinine clearance

Lipids: MDA-TBARS $(\mathrm{nmol} / \mathrm{mL})$

Anti-oxidants

Vitamin $\mathrm{E}(\mu \mathrm{mol} / \mathrm{L})$

Thiol levels $(\mu \mathrm{mol} / L)$

1

1

1

2

1

1

1

2

1

1

6

1

3

2

2

1

1

1

1

1

1

This article is protected by copyright. All rights reserved. 


\begin{tabular}{|l|l|}
\hline Uterine artery blood flow: umbilical artery pulsatility index & 1 \\
\hline 6 Fetal or neonatal mortality & \\
\hline Death of neonate before discharge from hospital & 1 \\
\hline Perinatal mortality & 1 \\
\hline 7 Fetal outcomes & 1 \\
\hline Gestational age at delivery: unclear $^{\text {a }}$ & 1 \\
\hline Birth weight, g & 1 \\
\hline $\begin{array}{l}\text { Respiratory distress syndrome: supplemental oxygen }>24 \text { h and } \\
\text { radiographic findings }\end{array}$ & \\
\hline 9 Childhood outcomes & 1 \\
\hline Neurologic development: Prechtl neonatal neurologic examination score & 1 \\
\hline 10 Fetal biological indices & 1 \\
\hline Fetal heart rate: unclear ${ }^{\text {a }}$ & 1 \\
\hline Umbilical artery blood flow: umbilical artery pulsatility index & 1 \\
\hline
\end{tabular}

Abbreviations. BP, blood pressure; ACOG, American College of Obstetricians and Gynecologists;

HELLP, hemolysis, elevated liver enzyme levels, and low platelets; AST, aspartate aminotransferase;

ALT, aanine aminotransferase, MDA-TBARS, malondialdehyde thiobarbituric acid reactive substances.

${ }^{\text {a }}$ No outcome measure, definition, or instrument stated.

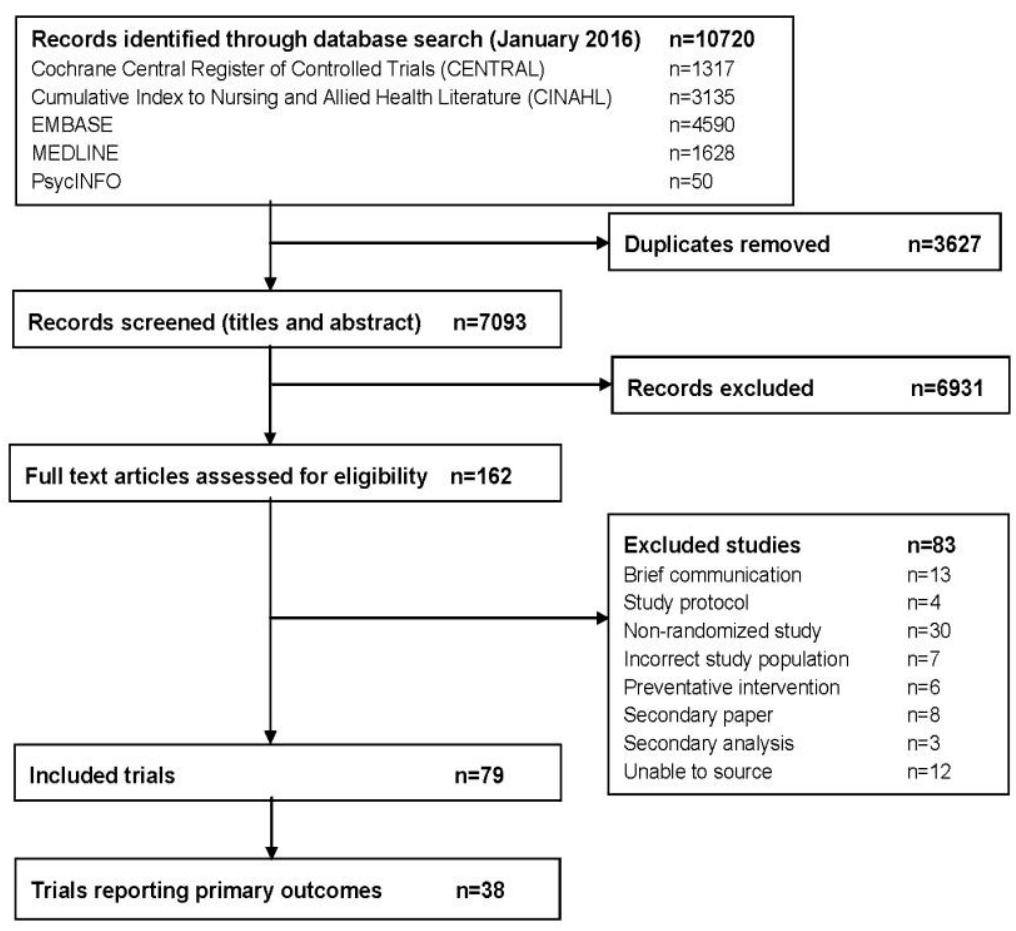

This article is protected by copyright. All rights reserved. 